\title{
Tevatron QCD physics
}

\author{
Robert Hirosky ${ }^{* *}$ \\ University of Virginia \\ E-mail: Hirosky@Virginia.Edu
}

The Run 2 Tevatron program at Fermilab is producing a wide range of QCD results, confronting new corners of the Standard Model with precision data, exploring the limits of perturbative theories and non-perturbative models, and performing direct searches for evidence of new physics. This talk summarizes recent results presented by the CDF and DØ Collaborations.

2008 Physics at LHC

September 29 - 4 October 2008

Split, Croatia

\footnotetext{
* Speaker.

${ }^{\dagger}$ For the CDF and DØ Collaborations.
} 


\section{Overview}

This talk summarizes recent QCD physics results from the CDF and DØ experiments at Fermilab, corresponding to integrated luminosities of up to $2 \mathrm{fb}^{-1}$ for $p \bar{p}$ collisions at $\sqrt{s}=1.96 \mathrm{TeV}$. The Tevatron QCD measurements confront Standard Model processes with unprecidented precision and are providing some of the most stringent limits on possible new physics scenarios.

\section{High $p_{T}$ jets}

The Tevatron data provide unique and increasingly precise measures of the strong interaction at large values of Björken-x and momentum exchange $\left(Q^{2}\right)$ in parton-parton interactions. Both $\mathrm{CDF}$ and $\mathrm{D} \emptyset$ have measured the differential cross sections for inclusive jet production as a function of jet transverse momentum $\left(P_{T}\right)$ in various ranges of jet rapidity $(y)$ [1], [2]. The DØ measurement is shown in Fig. 1. The smooth curves are predictions from NLO perturbative QCD, using the CTEQ6.5M parton distributions [3]. Also included are non-perturbative corrections for contributions due to the underlying event and hadronization effects calculated using PYTHIA [ [ and tune QW [5] for the underlying event model. Figure 2 shows the ratios of the DØ data to theory in the various rapidity regions. The shaded bands show systematic uncertainties on the data. The uncertainty in the theory, determined by varying the factorization and renormalization scales $\left(p_{T} / 2<=\mu_{R}, \mu_{F}<=2 p_{T}\right)$ is approximately $10-15 \%$. Of particular note in this figure is that the experimental uncertainties in the measurement are substantially less than those from the input PDFs, indicating that the Tevatron data will offer significant constraints on future PDF models. These data are included in a recent analysis [6] by the MSTW group and are leading to a significant reduction of their high- $x$ gluon distribution in the global analysis. The value of the Tevatron data for constraining PDF models remains substantial well into the LHC era. While a relatively larger percentage of high- $x$ events are gluon initiated at the LHC, the spectrum of parton momentum fractions is significantly softened at LHC energies. If one compares jet spectra using the invariant measure $x_{T}=2 * p_{T} / \sqrt{s}$, the Tevatron cross sections are approximately 100 times higher for all $x_{T}$ and 200 times higher for $x_{T}>0.5$. Effectively the LHC would need to acquire an integrated luminosity of around $1600 \mathrm{fb}^{-1}$ to match the same partonic integrated luminosity at high $x_{T}$ as the Tevatron will reach with $8 \mathrm{fb}^{-1}$.

$\mathrm{D} \emptyset$ has presented the first measurement of dijet angular distributions for dijet masses exceeding $1 \mathrm{TeV} / \mathrm{c}^{2}$. Dijet angular distributions are measured in terms of the variable $\chi_{\text {dijet }}=\exp \left(\mid y_{1}-\right.$ $y_{2} \mid$ ). At leading order in dijet production this is related to the partonic center of mass scattering angle via as: $\chi_{\text {dijet }}=\frac{1+\cos \theta^{*}}{1-\cos \theta^{*}}$. Rutherford scattering produces a flat $\chi$ distribution, Standard Model QCD is relatively flat, while many new physics signatures produce signals more strongly peaked at low values of $\chi$. Figure 3 shows normalized differential $\chi_{\text {dijet }}$ distributions $\left(\frac{1}{\sigma} \frac{d \sigma}{d \chi_{d i j e t}}\right)$ measured by $\mathrm{D} \varnothing$ in various ranges of the invariant mass of the dijet system. Predictions for Standard Model QCD processes are shown in the solid line. Also shown are expectations for various new physics scenarios. Observing good agreement between Standard Model expectations and the data, limits are set in several new physics scenarios. These are summarized in Table 1. A CDF dijet analysis compares the measured dijet mass spectrum to a smooth ansatz function (see Fig. ఫ). In this analysis a "bump hunt" is performed to search for indications of resonant structures in the dijet 


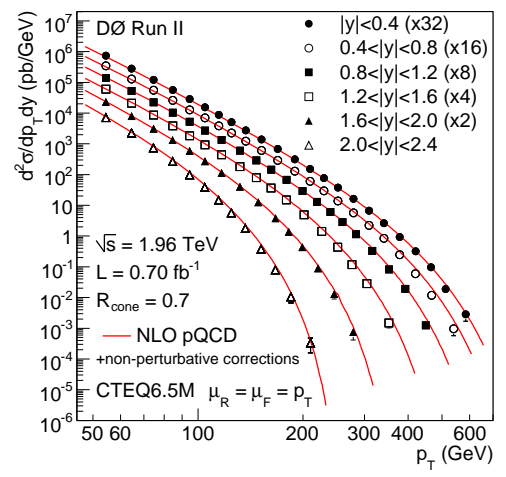

Figure 1: $D \emptyset$ inclusive jet cross section versus $P_{T}^{\text {jet }}$ measured in bins of $\left|\eta^{j e t}\right|$ 佨. The solid lines represent NLO PQCD predictions with nonperturbative corrections.

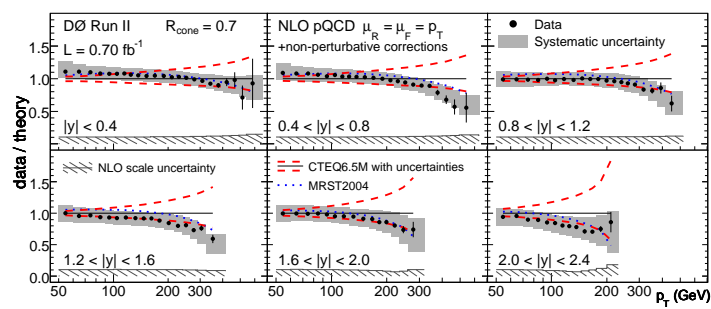

Figure 2: Data divided by theory for the DØ inclusive jet cross section measurements [2]]. Systematic uncertainties on the data are shown by shaded bands. NLO PQCD scale uncertainties are shown at the bottom of each panel. Solid(dashed) lines represent the cental prediction(error band) using the CTEQ6.5 PDF set. The predictions using MRST2004 PDFs is shown by the dotted lines.

mass spectrum. No significant resonances are observed and this analysis proceeds to determine exclusion regions for various new physics scenarios. A sampling of these is given in Table 1 .

\begin{tabular}{|c|c|c|c|c|c|c|}
\hline & $\begin{array}{c}\text { composite } \\
\text { quarks } \\
\text { [D] }\end{array}$ & $\begin{array}{c}\mathrm{TeV}^{-1} \\
\text { extra } \\
\text { dims. } \\
{[8]}\end{array}$ & $\begin{array}{l}\text { A.D.D. } \\
\text { large } \\
\text { extra } \\
\text { dims. [9] }\end{array}$ & $\begin{array}{l}\text { excited } \\
\text { quark } \\
10\end{array}$ & $\begin{array}{l}\text { color octet } \\
\text { techni-rho } \\
\text { 111 }\end{array}$ & $\begin{array}{c}\text { axi-gluon } \\
\& \text { coloron } \\
\text { 12 }\end{array}$ \\
\hline & \multicolumn{3}{|c|}{ units of $\mathrm{TeV}$} & \multicolumn{3}{|c|}{ units of $\mathrm{GeV} / \mathrm{c}^{2}$} \\
\hline CDF Exclusions & & & & $280-840^{(1)}$ & $260-1100^{(1)}$ & $260-1250^{(1)}$ \\
\hline DØ Limits & $\begin{array}{c}\Lambda \\
2.58^{(1)}\end{array}$ & $\begin{array}{c}M_{S} \\
1.42^{(2)}\end{array}$ & $\begin{array}{c}M_{c} \\
1.56^{(3)}\end{array}$ & & & \\
\hline
\end{tabular}

Table 1: Examples of excluded regions and limits on new physics processes from the CDF and DØ dijet data. (1) Most stringent limits. (2) Best limits from a hadron collider. (3) Best limit from a single proces at a hadron collider.

\section{Photons (+jets)}

The measurement of photon production is of particular interest in understanding QCD processes. As opposed to partons that carry color, a photon produced via parton interactions can reach a detector unaltered by subsequent interactions, thus serving as a direct probe of the hard scattering process. Furthermore, with a sufficient theoretical framework, photons can be used as a direct probe of the momentum fractions carried by gluons in $p \bar{p}$ scattering. In these analysis a combination of calorimeter and track-based isolation requirements are typically applied to reduce backgrounds from photons produced via jet fragmentation. Both CDF and DØ have performed a variety of photon analyses in Run 2. Figure 5 shows the preliminary CDF measure of the differential cross section for inclusive photon production as a function of photon $p_{T}$. Both DØ and CDF measurements of this process are found to be in agreement for $20<p_{T}^{\gamma}<300 \mathrm{GeV} / \mathrm{c}$, however 


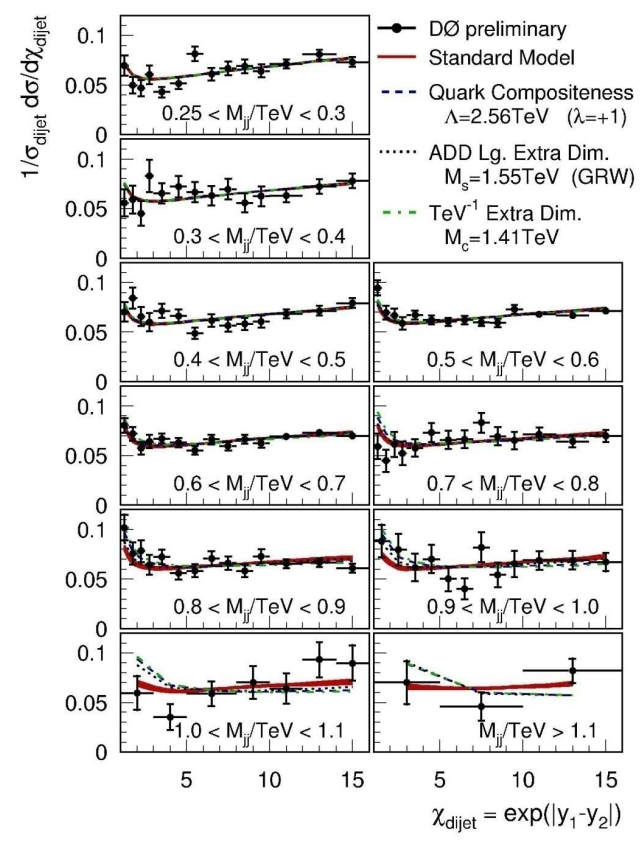

Figure 3: $\mathrm{D} \emptyset \chi_{\text {dijet }}$ distributions measured in ranges of dijet invariant mass. The solid line shows the expectation from Standard Model $\mathrm{QCD}$, also shown are expectations for several new physics scenarios: quark compositeness with $\Lambda=2.54 \mathrm{TeV}$ (dashed); ADD Large Extra Dimensions, $M_{s}=1.55 \mathrm{TeV}$ (dotted); and $\mathrm{TeV}^{-1}$ Extra Dimensions, $M_{c}=1.41 \mathrm{TeV}$ (dot-dash).
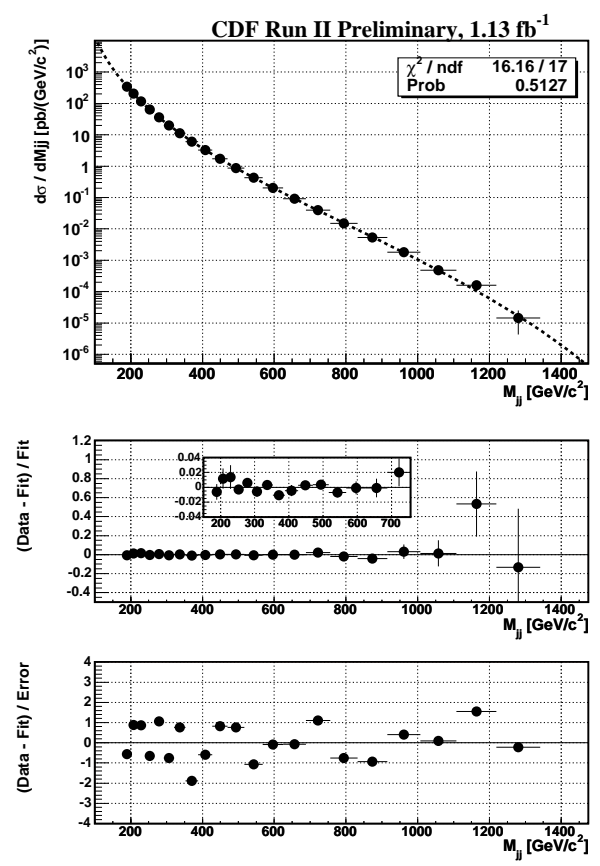

Figure 4: CDF dijet mass spectrum (top). Errors are statistical only. The spectrum is well represented by a smooth ansatz function (solid line). Comparing the fit to the data, no significant indication of resonant structure is observed (middle, bottom).

data and theory show shape differences approaching the lower $p_{T}$ range of the measurements. In order to better isolate kinematical regions that may contribute to a greater extent to the observed discrepancies with theory, $\mathrm{D} \emptyset$ has measured photon+jet production in regions subdivided by the locations of the leading photon and leading jet. Centrally produced photons are selected requiring $\left|y^{\gamma}<1.0\right|$ and event classes are defined with respect to the leading jet: central $\left(\left|y^{j e t}<0.8\right|\right)$, forward $\left(1.5<\left|y^{j e t}<2.5\right|\right)$ and same side $\left(y^{\text {jet }} \cdot y^{\gamma}>0\right)$ versus opposite side $\left(y^{\text {jet }} \cdot y^{\gamma}<0\right)$ pairs. Figure 6 shows the DØ measurements compared to NLO PQCD calculations.

Photon plus heavy flavor is an important signature in various extensions to the Standard Model, examples include Technicolor, SUSY, 4th generation fermions, and an excited b-quark. Photons plus b-flavor jets also serve as a probe of the b-content of the proton. With increasing data the Tevatron experiments are gaining access to observe photon plus heavy flavor processes. CDF has performed two measurements: a b-tagging analysis using their inclusive photon data set shown in Fig. 7 (top) and an analysis using a specially triggered data set using their silicon vertex detector to select events with a displaced vertex as well as a photon candidate, see Fig. 7 (bottom). Both analyses are in reasonable agreement with leading order predictions modeled by PYTHIA. DØ has a measurement of the the triple differential cross sections $d^{3} \sigma /\left(d p_{T}^{\gamma} d y^{\gamma} d y^{j e t}\right)$ for both $\gamma+c$ and $\gamma+b$ as a function $p_{T}^{\gamma}$. The results, shown in Fig. 8 , are in agreement with NLO PQCD predictions 


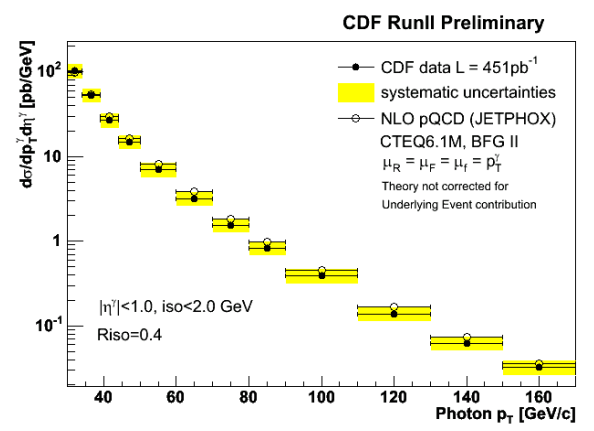

Figure 5: CDF measurement of inclusive photon production versus $p_{T}$, for $\left|\eta^{\gamma}\right|<1$.0. Data(theory) values are shown by the solid(open) symbols.

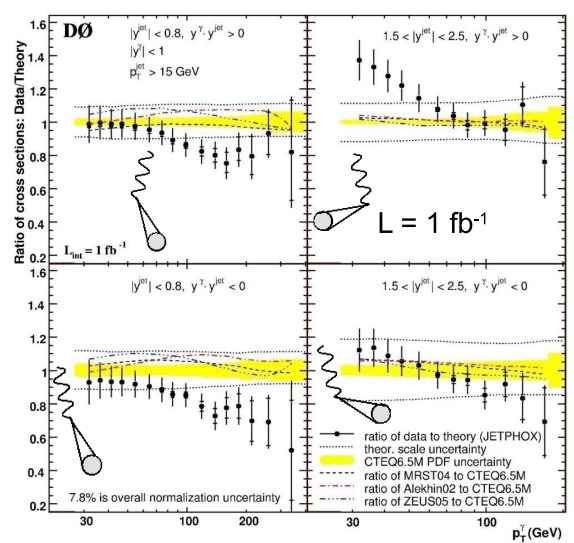

Figure 6: Data/theory ratios for the $\mathrm{D} \emptyset$ photon+jet measurements. Cross section ratios for photon+jets production are plotted versus $p_{T}^{\gamma}$ and further subdivided into kinematical regions based on the locations of the photon and leading jet.

over the full range of $\gamma+b$ data. In the case of $\gamma+c$ agreement is only observed for $p_{T}^{\gamma}<50 \mathrm{GeV}$ with an increasing divergence from predictions at higher $p_{T}$. This difference is somewhat reduced using a PDF model including intrinsic charm [13].

\section{4. $W / Z+$ jets}

$\mathrm{W} / \mathrm{Z}$ plus jet events are analogous to $\gamma$ plus jets in that the daughter particles from the vector boson decays yield a direct probe of the underlying dynamics of the parton scattering. These final states are also important signatures, as well as backgrounds, to a variety of Standard Model and new physics processes. Results from W/Z plus jet measurements at the Tevatron are covered in detail in S. Lammer's contributions to these proceedings. Here we discuss a single measurement of the $p_{T}$ spectrum of Z-bosons at $\mathrm{D} \emptyset$.

High $p_{T} \mathrm{Z}$ production is dominated by the associated emission of one or more hard partons. This process is well modeled in PQCD. For $p_{T}^{Z}$ around $30 \mathrm{GeV} / \mathrm{c}$ and lower at the Tevatron, the $\mathrm{Z}$ is primarily balanced by soft gluon emission. This process can be described by using gluon re-summation techniques. $\mathrm{D} \varnothing$ has performed a new measurement of the $p_{T}^{Z}$ to extract the main coefficient in the BLNY parametrization [14] as used in the RESBOS monte carlo. An earlier measurement [15] of the $p_{T}^{Z}$ spectrum was restricted by detector resolution, limiting the accuracy with which the observed spectrum could be used to extract model parameters. An updated analysis uses a new technique based on event quantities, primarily sensitive to angular resolutions of the detector, reducing overall systematic uncertainties in the analysis. The new analysis is performed for Z's decaying into either electron or muons pairs. Rather than measure $p_{T}^{Z}$ directly, it is instead decomposed into orthogonal components according to the event axis, $\hat{t}={\overrightarrow{p_{T}}}^{1}-{\overrightarrow{p_{T}}}^{2} /\left|{\overrightarrow{p_{T}}}^{1}-{\overrightarrow{p_{T}}}^{2}\right|$, where $p_{T}^{1,2}$ represent the individual lepton momenta. $\vec{p}_{T}^{Z}={\overrightarrow{p_{T}}}^{1}+{\overrightarrow{p_{T}}}^{2}$ is then decomposed into components transverse to the axis, $a_{T}=\left|\vec{p}_{T}^{Z} \times \hat{t}\right|$, and aligned with the axis, $a_{L}=\vec{p}_{T}^{Z} \cdot \hat{t}$. At low $p_{T}^{Z}$ the uncertainty on $a_{T}$ scales as the uncertainty on the individual lepton $p_{T}$ s multiplied by 

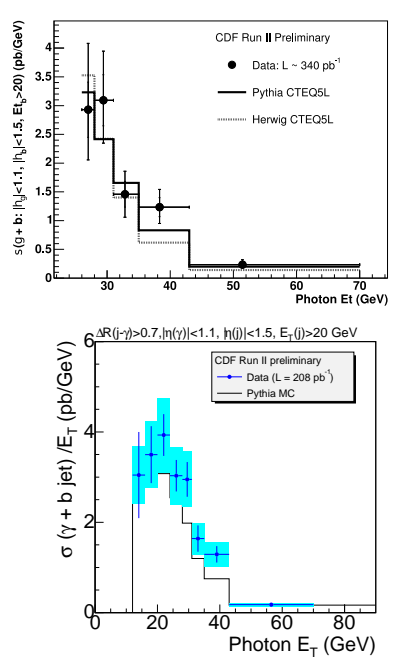

Figure 7: $\mathrm{CDF}$ measurements of $\gamma+\mathrm{b}$-jet production. Top: $\frac{d \sigma(\gamma+\mathrm{b}-\mathrm{jet})}{d E_{T}^{\gamma}}$ measured in inclusive $\gamma+$ jet sample with application of $b$-tagging. Bottom: The same measured using a specially triggered data sample requiring identification of a displaced vertex.
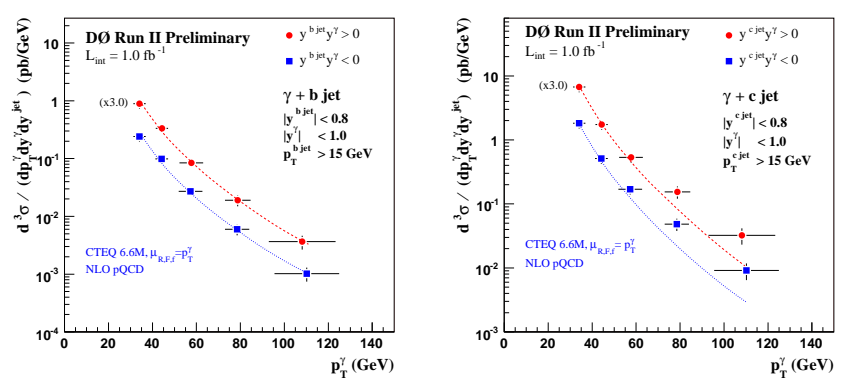

Figure 8: $D \emptyset$ measurements of the differential cross sections for $\gamma+\mathrm{b}$-jet (left) and $\gamma+\mathrm{c}$-jet (right) production, $\frac{d^{3} \sigma}{d p_{T}^{\gamma} d y^{\gamma} d y^{j e t}}$. Measurements (symbols) are shown in two kinematic regions, $y^{\text {jet }} \cdot y^{\gamma}>0$ and $y^{\text {jet }} \cdot y^{\gamma}<0$. Error bars include all uncertainties. The NLO theoretical predictions calculated using the CTEQ6.6M PDFs [16] are shown by the dotted lines.

the sine of a small angle. The technique is shown schematically in Fig. 9. Using this method $\mathrm{D} \emptyset$ extracts the single best measure of the value $g_{2}$ in the BLNY parametrization: $g_{2}=0.63 \pm$ $0.02(\exp ) \pm 0.03(P D F)$ [Preliminary].

\section{Diffractive physics and the underlying event}

As part of a program of diffractive physics measurements, CDF has performed a variety of analyses requiring a forward proton tag in downstream Roman-pot detectors. These include a recent measure of events with a $\mathrm{W}$ or $\mathrm{Z}$ boson produced via a diffracive process. Ultimately such measurements can lead to an improved understanding of the diffractive structure function. The ratios of diffractive to non-diffractive $\mathrm{W} / \mathrm{Z}$ production in the region of fractional momentum loss of the scattered proton $0.03<\xi<0.10$ and 4-momentum transfer squared $|t|<1$ are found to be $R_{W}=[0.97 \pm 0.05($ stat $) \pm 0.11($ syst $)] \%$ and $R_{Z}=[0.85 \pm 0.20($ stat $) \pm 0.11($ syst $)] \%$. Results from the $\mathrm{W}$ analysis are shown in Fig. 10.

CDF has presented studies of the event topology in Drell-Yan lepton-pair production in protonantiproton collisions at $1.96 \mathrm{TeV}$. The direction of the lepton-pair is used to define three regions of $\eta-\phi$ space on a event by event basis; toward, away and transverse to the vector sum of the lepton-pair. The transverse region is very sensitive to the underlying event. The data are corrected to the particle level and are then compared with the PYTHIA Tune AW. The properties of the underlying event are examined as a function of the transverse momentum of the leptonpair and the data are also compared with previous measurements of the underlying event in high 


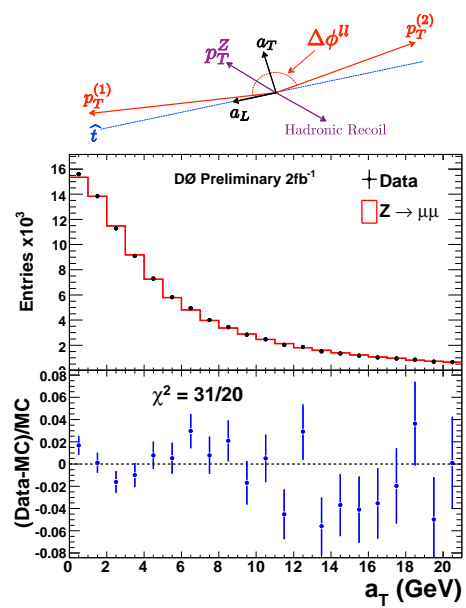

Figure 9: Top: Variables used in $\mathrm{D} \emptyset P_{T}^{Z}$ analusis. Bottom: The $a_{T}$ distribution for di-muon events. The solid line shows the prediction from RESBOS with a tuned value of the $g_{2}$ parameter.

transverse momentum jet production. The goal is to improve understanding and modeling of high energy collider events. The method studies charged particle $p_{T}$ densities in the above regions. In the away region, $p_{T}$ density increases with the $p_{T}$ of the lepton pair, whereas the $p_{T}$ density appears flat with respect to leption pair $p_{T}$ in the transverse or away regions. Results are summarized in Fig. 11

\section{Conclusion}

QCD analyses are a broad component of the CDF and $D \emptyset$ physics programs. As such, they are providing fundamental insights into light/heavy flavor and gluon PDFs. They are making increasingly sensetive statements about the interplay of perturbative and nonperturbative models and the limitations of possible new physics processes. Precise measures of benchmark processes at the Tevatron will also speed validation of any new physics signatures at the LHC. The full Tevatron data set will be $4-8$ times higher than most results in this talk. These data will dominate our models of the high-x gluon for some time and further improve our understading of rarer Standard Model processes well into the LHC era.

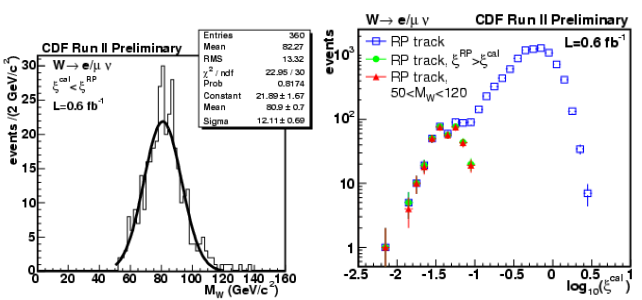

Figure 10: CDF Measurement of Diffractive W. Left: $M_{W}$ calculated using the Roman-pot track to reconstruct the kinematics of the neutrino. Right: The reconstructed $\xi$ distribution in the calorimeter for all events with a Roman-pot track, those consistent with a single interaction and missing energy due to a neutrino, and those with reconstructed $\mathrm{W}$ mass in the range of $50-120 \mathrm{GeV} / \mathrm{c}^{2}$.

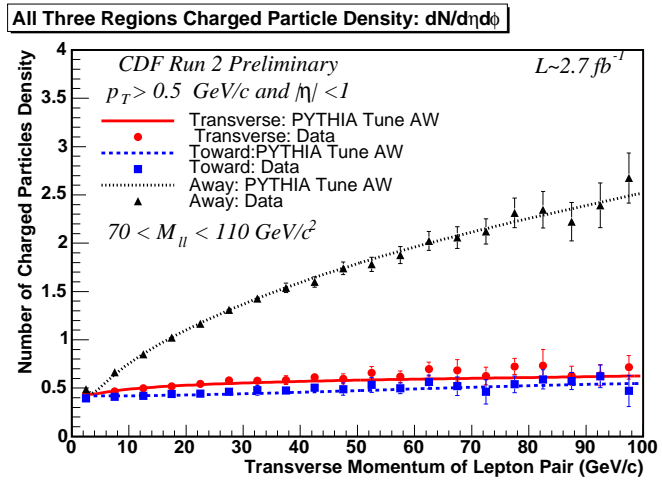

Figure 11: CDF measurement of the underlying event in Drell-Yan lepton-pair production. Charged particle multiplicity densities measured in three regions for electron and muon combined data (using tracks with $p_{T}>0.5 \mathrm{GeV} / \mathrm{c}$ and $|\eta|<1$ ). Lines represent PYTHIA Tune AW predictions. The data are corrected back to particle level (errors include both statistical and systematic uncertainty). 


\section{References}

[1] CDF Collaboration, Measurement of the inclusive jet cross section at the Fermilab Tevatron $p$ anti-p collider using a cone-based jet algorithm, Phys. Rev. D 78, 052006 (2008), [hep-ex/0512020].

[2] DØ Collaboration, Measurement of the Inclusive Jet Cross Section in p $\bar{p}$ Collisions at $\sqrt{s}=1.96 \mathrm{TeV}$, PRL 101, 062001 (2008), [arXiv.org:0802.2400].

[3] W.K. Tung et al., JHEP 0702, 053 (2007); J. Pumplin et al., JHEP 0207, 12 (2002); D. Stump et al., JHEP 0310, 046 (2003).

[4] T. Sjöstrand et al., Comp. Phys. Comm. 135, 238 (2001).

[5] R. Field in: M.G. Albrow et al. [TeV4LHC QCDWorking Group], arXiv:hep-ph/0610012.

[6] G. Watt, A. D. Martin, W. J. Stirling, R. S. Thorne, arXiv:0806.4890.

[7] E. Eichten, I. Hinchliffe, K. D. Lane and C. Quigg, Super Collider Physics, Rev. Mod. Phys. 56, 579 (1984) [Addendum-ibid. 58, 1065 (1986)]; P. Chiappetta and M. Perrottet, Possible bounds on compositeness from inclusive one jet production in large hadron colliders, Phys. Lett. B 253, 489 (1991); K. D. Lane, Electroweak and flavor dynamics at hadron colliders, arXiv:hep-ph/9605257.

[8] K. R. Dienes, E. Dudas and T. Gherghetta, Grand unification at intermediate mass scales through extra dimensions, Nucl. Phys. B 537, 47 (1999); A. Pomarol and M. Quiros, The standard model from extra dimensions, Phys. Lett. B 438, 255 (1998); K. Cheung and G. Landsberg, Kaluza-Klein states of the standard model gauge bosons: Constraints from high energy experiments, Phys. Rev. D 65, 076003 (2002).

[9] N. Arkani-Hamed, S. Dimopoulos and G. R. Dvali, The hierarchy problem and new dimensions at a millimeter, Phys. Lett. B 429, 263 (1998); D. Atwood, S. Bar-Shalom and A. Soni, Dijet production at hadron colliders in theories with large extra dimensions, Phys. Rev. D 62, 056008 (2000).

[10] U. Baur, I. Hinchliffe and D. Zeppenfeld, Int. J. Mod. Phys. A 2, 1285 (1987); U. Baur, M. Spira and P. M. Zerwas, Phys. Rev. D 42, 815 (1990).

[11] K. D. Lane and M. V. Ramana, Phys. Rev. D 44, 2678 (1991); E. Eichten and K. D. Lane, Phys. Lett. B 327, 129 (1994); K. Lane and S. Mrenna, Phys. Rev. D 67, 115011 (2003).

[12] J. L. Hewett and T. G. Rizzo, Phys. Rept. 183, 193 (1989).

[13] J. Pumplin et al., Phys. Rev. D 75, 054029 (2007).

[14] F. Landry et al., Phys. Rev. D 67, 073016 (2003).

[15] DØ Collaboration, Phys. Rev. L. 100, 102002 (2008).

[16] P. Nadolsky et al., Implications of CTEQ global analysis for collider observables, Phys. Rev. D78, 013004 (2008). 